\title{
Short Leg Film Is Sufficient for Postoperative Follow-Up in Total Knee Arthroplasty
}

\author{
David M. Fang, Tatsuya Sueyoshi, Kenneth E. Davis, Merrill A. Ritter \\ Center for Hip and Knee Surgery, Joint Replacement Surgeons of Indiana Research Foundation (JRSI), \\ Mooresville, USA \\ Email: jrsiresearch1@gmail.com
}

Received 5 June 2015; accepted 13 July 2015; published 16 July 2015

Copyright $(\underset{0}{ } 2015$ by authors and Scientific Research Publishing Inc.

This work is licensed under the Creative Commons Attribution International License (CC BY). http://creativecommons.org/licenses/by/4.0/

(c) (i) Open Access

\section{Abstract}

The gold standard for evaluating postoperative alignment after total knee arthroplasty (TKA) has been standing AP hip to ankle radiograph. The objective of this study was to determine if the angle between the anatomic axis of the tibia and femur measured on short standard radiographs accurately reflected overall coronal and mechanical alignment from full length knee films. Both full length and short length knee films were retrospectively compared measurements made on from 188 knees in 129 consecutive patients after primary TKA between July 1990 and January 1991. The tibiofemoral angle, distal femur and proximal tibia resection angles, and mechanical alignment were evaluated on the long leg by a blinded observer. The difference in the tibiofemoral angle measured on short and long leg films, $4.4(+/-2.9)$ vs $5.2(+/-3.0)$ degrees of valgus, respectively, was statistically significant $(p<0.01)$. The correlation coefficient between the tibiofemoral angle and mechanical axis was $0.9(p<0.01)$. There was no difference in the distal femur or proximal tibia resection angle measured on short length knee vs long leg films. In conclusion, the tibiofemoral angle measured on short length knee radiographs accurately reflects postoperative alignment. The differences between tibiofemoral angle and the mechanical alignment are highly correlated and equivalent measures of overall alignment.

\section{Keywords}

Total Knee Rthroplasty, Tibiofemoral Angle, Standing AP Radiograph, Accuracy

\section{Introduction}

Restoring coronal alignment in total knee arthroplasty (TKA) has been associated with improved implant survival [1] [2]. The "normal" alignment of the lower extremity has been described as a mechanical axis passing 
through the center of the knee and perpendicular to the ground, or an anatomic tibiofemoral angle of 7 to 9 degrees of valgus. Moreland emphasized the need for full length standing AP hip to ankle films $(14 \times 51$ inch) to assess postoperative alignment [3]. Peterson and Engh compared measurements from long and short films and reported a statistically significant difference of 1.4 degrees, but found that the mechanical alignment was strongly and directly related to the anatomic tibiofemoral angle [4]. Postoperative long leg films, however, are not routinely obtained because of the technical difficulty and increased cost. To determine the value of long leg films, the measurements from short standard length $(14 \times 17 \mathrm{inch})$ and long leg films are compared. The anatomic tibiofemoral angle was also compared with the mechanical axis (hip-knee-ankle angle) as a measure of overall coronal alignment.

\section{Methods}

Between July 1990 and January 1991, all primary TKAs were followed postoperatively with long leg and short knee films. Patients who did not have full length radiographs or more than 2 months of follow-up were excluded from the study. After IRB approval, the eligible 188 knees, 129 patients including 81 female and 48 males were studied (Table 1(a)). There were 170 osteoarthritis, 4 osteonecrosis, 10 rheumatoid arthritis and 10 other diagnosed knees. All cases utilized with Anatomic Graduate Components (AGC, Biomet, Inc, Warsaw, IN) total knee system (Table 1(b)). Long leg films were obtained 2 months after surgery using a standardized and previously described protocol [5] and evaluated by an independent, blinded observer. Several angles were measured to the nearest degree using a hand held goniometer as previously described, including the distal femoral and proximal tibia cut angles, and the tibiofemoral angle between the anatomic axis of the tibia. For short leg film, the tibiofemoral angle was acquired through the equation: (Femoral cut angle - 90) - (90 - tibial cut angle). The following measurements were made on short length films by the attending surgeons: the distal femur and proximal tibia resection angles.

Table 1. (a) Patient Demographics. (b) Diagnosis and Prosthesis type. OA: osteoarthritis, ON: osteonecrosis, RA: rheumatoid arthritis.

(a)

\begin{tabular}{lc}
\hline Knees & 188 \\
Patients & 129 \\
Female & 81 \\
Male & 48 \\
& \\
Average Age & 70.5 \\
Average BMI & 27.8 \\
\hline
\end{tabular}

(b)

\begin{tabular}{ccc|}
\hline Diagnosis & number & percent \\
\hline OA & 170 & $90.4 \%$ \\
ON & 4 & $2.1 \%$ \\
RA & 10 & $5.3 \%$ \\
Other & 4 & $2.1 \%$ \\
Total & 188 & $100 \%$ \\
\hline Prosthesis & number & percent \\
\hline AGC (all poly) & 138 & $73.4 \%$ \\
AGG (metal tibia) & 49 & $26.1 \%$ \\
Other & 1 & $0.5 \%$ \\
Total & 188 & $100.0 \%$ \\
\hline
\end{tabular}




\section{Results}

The mean tibial cut angle was $89.7( \pm 2.4)$ of varus on long length films and was $89.3( \pm 2.5)$ on short length films (Table 2). There was no significance in tibial cut angle and its standard deviation. The mean femoral cut angle measured on long length films was $95.5( \pm 2.3)$ of valgus, compared to $95.1( \pm 4.1)$ degrees of valgus as measured on short leg radiographs. There was no significance in the femoral cut angle, but there was a significant high standard deviation ( $\mathrm{p}<0.0001$ ) on short films, which means that the femoral angle is more than $200 \%$ variable on short films compared to long films. The mean tibiofemoral angle was $5.2( \pm 3.0)$ of valgus on long films, compared to $4.4( \pm 2.9)$ of valgus on short leg films. The difference of 0.8 degrees, however, was statistically significant $(\mathrm{p}=0.0005)$ (Table 2$)$. The tibiofemoral angle from short length films was found to be highly correlated with the mechanical alignment from long leg films, $r=0.9(p<0.01)$.

\section{Discussion}

The current study demonstrated the clinical usefulness of a short film in postoperative follow-up. Although the measurements of overall coronal alignment from long and short films were statistically different, the difference of less than one degree is not of significant importance clinically. This difference detected in this study of 0.8 degrees was similar in magnitude to previous reports. Dorr et al reported a difference of $1.9^{\circ}$ of valgus between long leg and standard knee films [6].

To use a long or a short film is an old and new problem in TKA [4] [7]-[9]. Some studies suggested the anatomic axis of the knee on a short film appears to be a valid alternative to the hip-knee-ankle axis of the limb on a long leg radiograph [7] [8]. Other study concluded short knee films cannot substitute for whole leg views when accurate assessment of the hip-knee-ankle axis is essential [9]. Peterson and Engh studied 50 patients with long leg and standard knee films and found a mean tibiofemoral angle of $5.8^{\circ} \pm 3.1^{\circ}$ of valgus, compared to $7.2^{\circ} \pm$ $3.9^{\circ}$ valgus on long leg films [4]. Their difference of $1.4^{\circ} \pm 2.2^{\circ}$ was similar to that found in this study, but was interpreted to be clinically significant based on the potentially large difference based on a wide confidence interval. However, the error associated with making measurements with a handheld goniometer as reflected by the standard deviation does not make this difference clinically significant. Rather, the fact that the standard deviation was greater than the actual detected difference only illustrates the lack of precision of the measurements and the small sample size.

Short length knee films underestimated the degree of valgus in the study, which agrees with the findings of Peterson and Engh [4]. Technical difficulties associated with accurately determining the anatomic axis of the tibia and femur may account for some of the differences between measurements made on short knee films versus long leg films. Even though the short length knee films were centered at the knee joint, the visualization of both

Table 2. Average measured angles, Std. dev; Standard deviation.

\begin{tabular}{cccc}
\hline & Tibia cut angle & Std. dev. & Range \\
\hline Long film & 89.7 & \pm 2.4 & {$[83.5,98.0]$} \\
Short film & 89.3 & \pm 2.5 & {$[82.0,96.0]$} \\
& $\mathrm{p}=0.0709$ (paired t-test) & $\mathrm{p}=0.4673$ (F test) & Range \\
\hline & Femoral cut angle & Std. dev. & {$[89.1,102.0]$} \\
\hline Long film & 95.5 & \pm 2.3 & {$[84.0,105.0]$} \\
Short film & 95.1 & $\mathrm{p}<0.0001$ (F test) & Range \\
\hline Long film & 0.1736 (paired t-test) & Std. dev. & {$[-4.0,13.0]$} \\
\hline Thort film & 5.2 & \pm 3.0 & {$[-2.0,13.0]$}
\end{tabular}


sides of the joint was not equal. The metaphyseal regions of the distal femur and proximal tibia may not be symmetric or collinear with the diaphysis. Bowing of the tibia and femur not visualized on the short length knee films could also lead to differences, but were not identified as a source of error in this study. Finally, although the method of obtaining hip to ankle films was standardized, several films were taken in external rotation. This illustrates the difficulty and additional error of using long leg films.

Mechanical alignment can only be determined on long leg films. In this study, the angle between the mechanical axis of the femur and tibia was found to be directly related to the anatomic tibiofemoral angle with a correlation coefficient of 0.9. The mechanical alignment can also be described as the distance of the mechanical axis of the lower extremity to the center of the knee. Peterson and Engh [4] showed that this measure was highly and directly correlated with the anatomic tibiofemoral angle, $\mathrm{p}<0.001$. Thus, these results show that either the mechanical or anatomic angle can be used to assess postoperative alignment of the lower extremity.

\section{Conclusion}

Postoperative alignment is one of the most important objective measures and determinants of success after TKA. The tibiofemoral angle measured on short length knee radiographs is equivalent to the mechanical alignment obtained on long leg films in assessing coronal alignment. The current study demonstrated a short leg film is sufficient for postoperative follow-up. Further study should reconfirm this application with digital films on computer display which are currently standard.

\section{References}

[1] Lotke, P.A. and Ecker, M.L. (1977) Influence of Positioning of Prosthesis in Total Knee Replacement. Journal of Bone and Joint Surgery (American), 59, 77-79.

[2] Ritter, M.A., Faris, P.M., Keating, E.M. and Meding, J.B. (1994) Postoperative Alignment of Total Knee Replacement: Its Effect on Survival. Clinical Orthopedics and Relatated Research, 467, 146-154. http://dx.doi.org/10.1097/00003086-199402000-00021

[3] Moreland, J.R. (1988) Mechanisms of Failure of Total Knee arthroplasty. Clinical Orthopedics and Relatated Research, 226, 49-64. http://dx.doi.org/10.1097/00003086-198801000-00010

[4] Petersen, T.L. and Engh, G.A. (1988) Radiographic Assessment of Knee Alignment after Total Knee Arthroplasty. Journal of Arthroplasty, 3, 67-72. http://dx.doi.org/10.1016/S0883-5403(88)80054-8

[5] Cates, H.E., Ritter, M.A., Keating, E.M. and Faris, P.M. (1993) Intramedullary versus Extramedullar Femoral Alignment Systems in Total Knee Replacement. Clinical Orthopedics and Relatated Research, 286, 32-39.

[6] Dorr, L.D., Conaty, J.P. and Schreiber, R. (1984) Technical Factors that Influence Mechanical Loosening of Total Knee Arthroplasty. In: Dorr, L., Ed., The Knee: Papers of the 1st Scientific Meeting of the Knee Society, University Park Press, Baltimore, 121.

[7] Hinman, R.S., May, R.L. and Crossley, K.M. (2006) Is There an Alternative to the Full-Leg Radiograph for Determining Knee Joint Alignment in Osteoarthritis? Arthritis \& Rheumatism, 55, 306-313. http://dx.doi.org/10.1002/art.21836

[8] Kraus, V.B., Vail, T.P., Worrell, T. and McDaniel, G. (2005) A Comparative Assessment of Alignment Angle of the Knee by Radiographic and Physical Examination Methods. Arthritis \& Rheumatism, 52, 1730-1735. http://dx.doi.org/10.1002/art.21100

[9] Issa, S.N., Dunlop, D., Chang, A., et al. (2007) Full-Limb and Knee Radiography Assessments of Varus-Valgus Alignment and Their Relationship to Osteoarthritis Disease Features by Magnetic Resonance Imaging. Arthritis \& Rheumatism, 57, 398-406. http://dx.doi.org/10.1002/art.22618 